\title{
Understanding cardiac systolic performance beyond left ventricular ejection fraction
}

\author{
Elena-Laura Antohi ${ }^{1,2}$, Ovidiu Chioncel ${ }^{1}(0)$ \\ ${ }^{1}$ ICCU and Cardiology $1^{\text {st }}$ Department, Emergency Institute for Cardiovascular Diseases “C.C.Iliescu”, 022328 Bucharest, Romania \\ ${ }^{2}$ University for Medicine and Pharmacy “Carol Davila”, 020021 Bucharest, Romania \\ *Correspondence: Elena-Laura Antohi, ICCU and Cardiology 1st Department, Emergency Institute for Cardiovascular Diseases \\ “C.C.Iliescu”, 022328 Bucharest, Romania; University for Medicine and Pharmacy “Carol Davila” Bucharest, Romania. laura. \\ antohi@gmail.com
}

Academic Editor: Akiko Mammoto, Medical College of Wisconsin, USA

Received: January 14, 2020 Accepted: April 17, 2020 Published: April 30, 2020

Cite this article: Antohi EL, Chioncel C. Understanding the cardiac systolic performance beyond left ventricular ejection fraction. Explor Med. 2020:1:75-84. https://doi.org/10.37349/emed.2020.00006

\begin{abstract}
Left ventricular ejection fraction is the critical parameter used for heart failure classification, decision making and assessing prognosis. It is defined as a volumetric ratio and is essentially a composite of arterial and ventricular elastances, but not intrinsic contractility. The clinician should be aware of its numerous limitations when measuring and reporting it. And make a step toward more insightful understanding of hemodynamics.
\end{abstract}

\section{Keywords}

Left ventricular ejection fraction, ventriculo-arterial coupling, left ventricular functions, hemodynamics

\section{Introduction}

Left ventricular ejection fraction (LVEF) is the critical parameter used heart failure (HF) [1]. Today, more than 50 years after the first reports describing it [2], LVEF likely remains the most-often used parameter to assess LV systolic performance, because of the simplicity of its calculation by whatever non-invasive imaging method, the ease of understanding it on the part of clinicians (cardiologist or otherwise), and its relevance to clinical decision-making and prognosis. Its core clinical value is to define HF with reduced ejection fraction (HFrEF) that triggers the use of neurohormonal medications that improve outcomes [1].

The clinician should consider its limitations and whenever possible, make use of other parameters to further characterize the hemodynamics of patients with heart disease.

\section{LVEF definition}

LVEF represents the proportion of diastolic volume ejected during ventricular contraction and is calculated as a dimensionless ratio between stroke volume (SV) and end-diastolic volume (EDV) [3]. 


\section{LVEF measurement}

LVEF calculation is possible using invasive angiographic methods (which used to be considered the gold standard) or by widely available non-invasive imaging. Each technique has its draw-backs and sources of error, and there are differences in methodologies and algorithms.

Most experts calculate LV volumes based on the geometrical approximation of the normal ellipsoid shape of the $\mathrm{LV}$, which becomes a source for error when $\mathrm{LV}$ remodeling in the context of eccentric hypertrophy (i.e. mode M derived LVEF [4] or the area-length method). Two-dimensional (2D) echocardiographic calculation of the LVEF using the recommended Simpson method of disks [3] is easy to perform, using widely available echo machines; nonetheless accuracy suffers from image quality.

These issues have led to the development of less geometrical methods and techniques which rely on good border delineation, including three-dimensional (3D) echocardiography or computer tomography (CT). On the other hand, improvement of endocardial border delineation with contrast 2D echocardiography has been shown to derive more reproducible values [5], more similar to cardiac magnetic resonance (CMR).

There is generally good agreement between methods, as described in Table 1 [5-10], even though the same studies showed that LV volumes tended to be smaller when compared to CMR imaging and had significant variability. The same applies for radionuclide techniques: in a large community study, Single-Photon Emission Computed Tomography (SPECT) did not correctly discern between the left atrium and LV, yet the sensitivity and specificity for determining LVEFs of $<40 \%$ were $90 \%$ and $86 \%$, respectively [11].

Table 1. Comparison of LVEF as measured by different non-invasive imaging methods, reported mean difference and agreement

\begin{tabular}{|c|c|c|c|c|}
\hline & Echo 3D vs. CMR & SPECT vs. CMR & MDCT vs. CMR & Echo3D vs. SPECT \\
\hline Mannaerts et al. 2003 [6] & MD $1.8 \% \pm 11.6 \%$ & & & \\
\hline Persson et al. 2005 [7] & & MD $4 \% \pm 7 \%(P<0.001)$ & & \\
\hline \multirow[t]{2}{*}{ Belge et al. 2006 [8] } & & & MD $1 \% \pm 21 \%(P=N S)$ & \\
\hline & & & $r=0.95 ; P<0.001$ & \\
\hline Soliman et al. 2008 [9] & MD $0.2 \% \pm 6.2 \%$ & & & \\
\hline \multirow[t]{3}{*}{ Beitner et al. 2019 [10] } & MD $0.56 \% \pm 15 \%$ & MD $0.75 \% \pm 10 \%$ & & $1.3 \% \pm 20 \%$ \\
\hline & ICC 0.71 & ICC 0.89 & & ICC 0.51 \\
\hline & $\beta=0.72, P=0.003$ & $\beta=0.90, P<0.001$ & & \\
\hline
\end{tabular}

Echo2D: bidimensional echocardiography; Echo3D: three dimensional echocardiography; MDCT: gated multidetector row computed tomography; MD: mean difference; NS: not significant; r: correlation index; ICC: intraclass correlation coefficient

CMR has become the new gold standard, against which all methods are compared, due to the excellent image resolution and thus accurate border detection, good reproducibility, low test-retest variation [10-12].

Nonetheless, echocardiography can correctly identify patients with depressed and non-depressed LVEF $[5,11]$. Echocardiographic calculation of stroke volume by measuring the velocity time integral in the absence of left-sided regurgitation can overcome the difficulties of delineating trabeculations in a small LV cavity at end-systole.

\section{Normal range of $55 \%$ to $65 \%$ for $\mathrm{LVEF}$}

Defining the normal range of values is an overlooked issue in clinical practice.

First, the aforementioned differences in methodology and algorithms between diagnostic modalities make a fixed value unlikely; Wood et al. [5], in a 2014 analysis, observed that the reference values were based on studies involving cohorts as low as 60 patients ranging up to 1,200 , suggesting that further validation studies are required.

Second, abnormal LVEF values were clearly defined below 50\%; the dismal prognostic value of abnormal LVEF was observed in several cohorts [13-15]. Asymptomatic left ventricular systolic dysfunction defined as $\mathrm{LVEF}<50 \%$, although uncommon (up to $2 \%$ [14]), was an early marker of evolution toward severe congestive HF and death, nearly five-fold higher than in control subjects in the multi-ethnic study of atherosclerosis study [14]. 
The borderline gray zone between $50 \%$ and $55 \%$ has been considered the lower range of normal values. A recent study by Tsao et al. [16], showed that persons with LVEF of $50 \%$ to $55 \%$ in the community have greater risk for morbidity and mortality relative to persons with LVEF $>55 \%$. This led Fonarow and Hsu to question the paradigm and suggest that LVEF of $50 \%$ to $55 \%$ should not be considered within the "normal" range [17].

Third, there are recognized age, gender, and ethnicity differences. CMR data showed that women have higher LVEF than men in the general population, with higher SV for a given EDV, independent of known potential confounders (low LVEF defined as below the $2.5^{\text {th }}$ percentile of a healthy subset was $61 \%$ in women and 55\% in men) [18]; current guideline recommendations describe separate cutoffs [3]. A very large echocardiographic study in more than 8,000 healthy volunteers $<70$ years suggested ethnic-specific cutoffs for EF normal ranges [19]. LVEF was also shown to increase with age [20, 21], with significant difference between the extreme age groups, more pronounced in females (as demonstrated in one large population study $62 \% \pm 0.5 \%$ [age $7-20$ years] vs. $65 \% \pm 0.3 \%$ [age $60-80$ years] [20]).

A fourth issue involves the physiologic variations of LVEF.

During exercise, increase LVEF was demonstrated. Athletes express lower rest EF values $(52.9 \% \pm 5.86 \%$ compared to non-athletic controls $58.2 \% \pm 6.05 \%, P<0.001$ ), but with more significant $\triangle \mathrm{LVEF}$ increase of $16.5 \% \pm 8.51 \%$ during exercise and with greater $\mathrm{LV}$ contractility reserve $v s$. non-athletes [22].

There is also evidence for circadian and sleep-induced LVEF changes. A small study in the 1990s identified a temporary (up to $30 \mathrm{~min}$ ) dramatic decrease of scintigraphy-measured LVEF at waking up in the morning, to as low as $<30 \%$ in some normal subjects [23]. The observation was not further investigated; however, given the recognized circadian changes in all other hemodynamic determinants of cardiac performance, it is to be regarded as noteworthy.

Large population studies show a Gaussian distribution of LVEF, with a mean value around $62 \%$ (measured by echo or CT), which probably ideal $[20,24]$. Another recently published analysis of a very large clinical dataset (403, 977 echocardiograms from 203, 135 unique patients), emphasizes the presumably "physiological" range of 60-65\%, which is associated with best survival; it also suggests the existence of a supra-normal LVEF phenotype associated with reduced survival [25].

\section{LVEF is not an intrinsic contractility parameter}

LVEF is regarded as an essential measure of LV systolic function [3], even though mathematical reasoning and early experimental work [26] have shown it is load-dependent.

Nonetheless, early experimental work on the sarcomere length-tension curve and Starling's law for heart contraction revealed a good correlation between sarcomere performance and EF [27]. More recent studies of structural and functional reverse remodeling reveal good correlation between changes in gene expression and LVEF $[28,29]$, more so than to other hemodynamic parameters, thereby demonstrating the ability of LVEF to reflect the contractility state of the myocardium [30].

In the search of a more integrative hemodynamic perspective, cardiac physiologists turned toward analysis of pressure-volume loops (PVL). This provides the basis for the calculation of arterial (Ea) and ventricular (Ees) elastances. Their ratio (Ea/Ees) represents the ventriculo-arterial coupling (VAC); its diagnostic and prognostic value has recently been recognized once more, in a consensus document of the Heart Failure Association [31]; this is particularly relevant for HFrEF.

Although accurate measurements require invasive techniques (methods and updated clinical utility are briefly described in a review by Bastos et al. [32]), Chen et al. [33], and Shishido et al. [34], introduced the "single-beat" Ees estimation derived from non-invasive studies.

The mathematical formulas are complicated; however, current widespread technology can compute these easily (including the iPad App simulation of PVL [35]) and could gain clinical utility [36].

Kass et al. [37], and Robotham et al. [38], have published on the relationship between LVEF and Ea and Ees, defining EF by the following formula: LVEF = Ees $[1-($ Vo/EDV $)] /($ Ea + Ees $)$, where Vo = the volume intercept at $0 \mathrm{mmHg}$ pressure [37]. 
It becomes understandable that LVEF results from permanent cross-talk between Ees and Ea, being subordinated to the VAC and explaining the lesser sensitivity of the LVEF to contractility itself [39]. Although, not a strong contractility discriminator, when regarded as a composite result of Ees and Ea, LVEF is a powerful predictor [39]. By mathematical consequence, for a considerable increase of the contraction force, a mild increase in Ea demonstrates a net blunting effect over the final LVEF augmentation, LVEF being thus predominantly afterload-dependent, especially with decreased contractility [39]. Experimental work already confirmed this $[37,40]$. Therefore, the understanding of left ventricular contractility should not rely only on the calculation of LVEF.

As pointed out by Kass et al. [37], in a pivotal article, PVL analysis allows for a more complete characterization of pump performance, separating loading factors from ventricular properties and, relevant for critical care practice, it enables the prediction of stroke volume and stroke work response to various interventions. In this context, Ees was adopted as an index of intrinsic LV contractility [41, 42]; it remains imperfect, being a time-varying non-linear function derived from single-point steady state formulas.

For patients with $\mathrm{HF}$ with preserved $\mathrm{EF}$ (HFpEF), EF cannot be discriminatory enough for the severity of the myocardial disease; the same is true for VAC as a dimensionless index alone; the integrated analysis of the parameters relating to structural changes and describing the coupling of $\mathrm{LV}$ and arterial system, including the absolute values of Ea, arterial compliance, Ees, Vo (Vo is the theoretical volume intercept at $0 \mathrm{mmHg}$ pressure and correlates as well to contractile function [43]), end-diastolic volume could further differentiate the severity of hypertrophied LV when facing high afterload (e.g., hypertension or aortic stenosis) [44-46].

The influence of heart rate on PVL is often neglected; it influences cardiac contractility by the forcefrequency relation; however, it is also a major contributor to Ea, as shown by Segers et al. [47], in a mathematical heart-arterial interaction model.

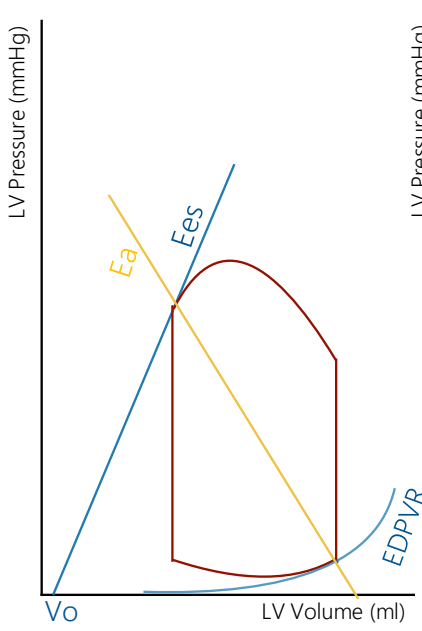

A. PVL at rest

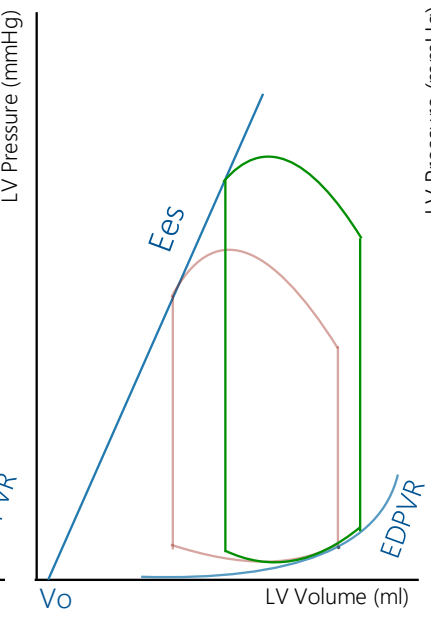

B. PVL with increase in afterload

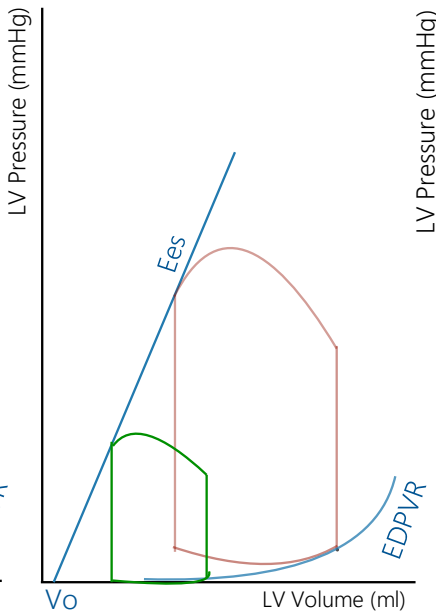

C. PVL with decrease in preload.

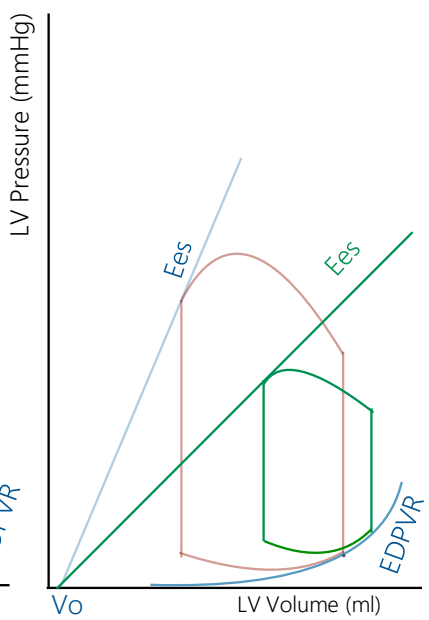

D. PVL with decrease in inotropy.

Figure 1. Various interventions on the PVL loop. A. LV PVL at rest; B. Increase in afterload (the PVL changes to the right and upwards); C. Decrease in preload (the PVL changes to the left and downwards); D. Decrease in inotropism (the PVL will change downwards and to the left)

An important contractility determinant is LV geometry. The variations in LV shape and size that occur with pathological remodeling, reflected by the LV volumes [both end-systolic (ESV) and EDV], decrease contractile efficiency and predict outcome [44, 48]. ESV is one of the essential components of Ees and VAC, while having a non-linear relationship with EF, as described in pivotal work by Kerkhof et al. [49, 50], on the volume regulation graph (VRG). Any change in the inotropic state will impact ESV [49, 50]. From this premises, Kerkhof [44] and Mann et al. [48], propose plotting LVEF as a function of end-systolic volume index, for a more accurate description of $\mathrm{LV}$ working point and improved classification of HF.

Monge García et al. [40], also acknowledge the limitations of LVEF as a contractility discriminant and propose an afterload (Ea)-adjusted LVEF; using the square root of Ea would account for the greater impact 
of afterload on VAC at low levels of contractility and significantly improve the ability to track LV contractility: LVEF Ea $=$ [square root of (PesXSV)] / EDV [40], which is not dimensionless, in contrast to the traditional EF.

PVL and the derived VRG could further deepen the hemodynamic understanding of LV systolic function (Figure 1 depicts various interventions on the LV and the subsequent change in PVL).

\section{Searching for other contractility indices}

There has been a consistent search for contractility parameters other than LVEF.

The maximum rate of left ventricular pressure rise (dP/dt) and its relation to EDV as described by Little [51] was very sensitive to inotropic stimulation, independent of the load.

Tissue Doppler Imaging (TDI) has been used for the past 30 years and validated as a sensitive tool for subclinical detection of longitudinal dysfunction for both left and right ventricles [52, 53].

Seo et al. [54], in an animal study the $S^{\prime}$ wave and LVEF against $L V \mathrm{dP} / \mathrm{dt}$ : $\mathrm{S}^{\prime}$, reflecting both LV longitudinal shortening and torsional deformation proved to be a more sensitive index of global LV contractility than LVEF, as it had a much stronger correlation to $\mathrm{dP} / \mathrm{dt}$ than $\operatorname{LVEF}(\mathrm{r}=0.665 \mathrm{vs} . \mathrm{r}=0.40$, respectively).

Beyond the measurement of TDI wave amplitude, precise time interval measurement is sometimes a neglected advantage. Myocardial performance index (MPI), derived by measuring systolic and diastolic time intervals using TDI M-mode through the mitral valve, was significantly associated with invasive (dPmax/dt) and non-invasive ( $\mathrm{LV}$ global longitudinal strain and strain rate) measures of LV systolic function; conventional measurement of MPI (using pulsed wave in the left ventricular outflow tract) was less reliable [55].

The superiority of myocardial deformation parameters (strain, strain rate) in risk assessment and as sensitive contractility indices have extensively been reviewed whenever the limitations of LVEF as a contractility marker are discussed $[12,56]$ and are now integrated into the essential recommendations for the echocardiographic assessment of LV function [3].

No less significant is the evaluation of $L V$ contractile/flow reserve (as the ability to augment its performance or provide adequate $\mathrm{SV}$ augmentation according to inotropic stimulation) [39, 57]. SV augmentation during dobutamine stress echo was found to be a powerful predictive parameter for good surgical outcomes in very high-risk surgery whether undergoing LV remodeling procedures [58] or aortic valve replacement [59].

The most important clinical limitation of LVEF is its inability to describe the HFpEF population. The hemodynamics of these patients is presumably better described using the VAC (which has normal or lower than normal values), and even more so by the absolute values of Ees (which are generally elevated) and the correspondingly abnormally elevated value of Ea at rest [60].

The interaction of the $\mathrm{LV}$ with the other cardiac chambers is equally important. Left atrial enlargement due to pressure and volume overload causes structural changes in the other chambers, including concomitant tricuspid annulus dilation, increased mobility of the tricuspid leaflets tricuspid regurgitation, and decreasing $\mathrm{RV}$ performance. This issue was addressed in detail by Zakir et al. [61], who demonstrated the correlation of LV diastolic function with RV systolic dysfunction, based on the invasive measurement of the pulmonary venous system. LV diastolic dysfunction causes increased left atrial filling pressure that can be transmitted backward, leading to pulmonary arterial hypertension and RV pressure overload. Brand et al. [62], demonstrated that systolic right heart function is significantly altered in patients with diastolic dysfunction and enlarged left atrium.

\section{Conclusion}

Although LVEF is essentially a volumetric index, it remains a powerful clinical parameter of LV myocardial function that represents the interplay between $\mathrm{LV}$ contractile state and the arterial afterload. To be able to adequately utilize it in clinical practice, the clinician should acknowledge its limitations as a contractility marker, the pitfalls that occur with measurement for the various imaging techniques and methods, and the significance of normal values. EF, can only provide limited information on cardiac performance; therefore, a more in-depth understanding of hemodynamics should always be sought. 


\section{Abbreviations}

CMR: cardiac magnetic resonance

CT: computer tomography

$\mathrm{dP} / \mathrm{dt}$ : maximum rate of left ventricular pressure rise

Ea: arterial elastance

EDV: end-diastolic volume

Ees: ventricular elastance

EF: ejection fraction

ESV: end-systolic volume

HF: heart failure

HFpEF: HF with preserved EF

HFrEF: HF with reduced EF

LV: left ventricle

LVEF: left ventricular ejection fraction

MPI: myocardial performance index

Pes: end-systolic pressure

PVL: pressure volume loop

SPECT: single-photon emission computed tomography

SV: stroke volume

VAC: ventriculo-arterial coupling

Vo: volume axis intercept for $0 \mathrm{mmHg}$

VRG: volume regulation graph

\section{Declarations}

\section{Author contributions}

ELA wrote the first draft of the manuscript. All authors contributed to manuscript revision, read and approved the submitted version.

\section{Conflicts of interest}

The authors declare that they have no conflicts of interest.

\section{Ethical approval}

Not applicable.

\section{Consent to participate}

Not applicable.

\section{Consent to publication}

Not applicable.

Availability of data and materials

Not applicable.

\section{Funding}

Not applicable. 


\section{Copyright}

(C) The Author(s) 2020.

\section{References}

1. Ponikowski P, Voors AA, Anker SD, Bueno H, Cleland JGF, Coats AJS, et al. 2016 ESC guidelines for the diagnosis and treatment of acute and chronic heart failure: the task force for the diagnosis and treatment of acute and chronic heart failure of the European Society of Cardiology (ESC) developed with the special contribution of the Heart Failure Association (HFA) of the ESC. Eur Heart J. 2016;37:2129-200.

2. Folse R, Braunwald E. Determination of fraction of left ventricular volume ejected per beat and of ventricular end-diastolic and residual volumes. Experimental and clinical observations with a precordial dilution technic. Circulation. 1962;25:674-85.

3. Lang RM, Badano LP, Mor-Avi V, Afilalo J, Armstrong A, Ernande L, et al. Recommendations for cardiac chamber quantification by echocardiography in adults: an update from the American Society of Echocardiography and the European Association of Cardiovascular Imaging. Eur Heart J Cardiovasc Imaging. 2015;16:233-70.

4. Teichholz LE, Kreulen T, Herman MV, Gorlin R. Problems in echocardiographic volume determinations: echocardiographic-angiographic correlations in the presence of absence of asynergy. Am J Cardiol. 1976;37:7-11.

5. Wood PW, Choy JB, Nanda NC, Becher H. Left ventricular ejection fraction and volumes: it depends on the imaging method. Echocardiography. 2014;31:87-100.

6. Mannaerts HF, Van Der Heide JA, Kamp O, Papavassiliu T, Marcus JT, Beek A, et al. Quantification of left ventricular volumes and ejection fraction using freehand transthoracic three-dimensional echocardiography: comparison with magnetic resonance imaging. J Am Soc Echocardiogr. 2003;16:101-9.

7. Persson E, Carlsson M, Palmer J, Pahlm O, Arheden H. Evaluation of left ventricular volumes and ejection fraction by automated gated myocardial SPECT versus cardiovascular magnetic resonance. Clin Physiol Funct Imaging. 2005;25:135-41.

8. Belge B, Coche E, Pasquet A, Vanoverschelde JL, Gerber BL. Accurate estimation of global and regional cardiac function by retrospectively gated multidetector row computed tomography: comparison with cine magnetic resonance imaging. Eur Radiol. 2006;16:1424-33.

9. Soliman OI, Kirschbaum SW, van Dalen BM, van der Zwaan HB, Mahdavian Delavary B, Vletter WB, et al. Accuracy and reproducibility of quantitation of left ventricular function by real-time three-dimensional echocardiography versus cardiac magnetic resonance. Am J Cardiol. 2008;102:778-83.

10. Beitner N, Jenner J, Sorensson P. Comparison of left ventricular volumes measured by 3DE, SPECT and CMR. J Cardiovasc Imaging. 2019;27:200-11.

11. Habash-Bseiso DE, Rokey R, Berger CJ, Weier AW, Chyou PH. Accuracy of noninvasive ejection fraction measurement in a large community-based clinic. Clin Med Res. 2005;3:75-82.

12. Marwick TH. Ejection fraction pros and cons: JACC state-of-the-art review. J Am Coll Cardiol. 2018;72:2360-79.

13. Verdecchia P, Angeli F, Gattobigio R, Sardone M, Porcellati C. Asymptomatic left ventricular systolic dysfunction in essential hypertension: prevalence, determinants, and prognostic value. Hypertension. 2005;45:412-8.

14. Yeboah J, Rodriguez CJ, Stacey B, Lima JA, Liu S, Carr JJ, et al. Prognosis of individuals with asymptomatic left ventricular systolic dysfunction in the multi-ethnic study of atherosclerosis (MESA). Circulation. 2012;126:2713-9.

15. Echouffo-Tcheugui JB, Erqou S, Butler J, Yancy CW, Fonarow GC. Assessing the risk of progression from asymptomatic left ventricular dysfunction to overt heart failure: a systematic overview and metaanalysis. JACC Heart Fail. 2016;4:237-48. 
16. Tsao CW, Lyass A, Larson MG, Cheng S, Lam CS, Aragam JR, et al. Prognosis of adults with borderline left ventricular ejection fraction. JACC Heart Fail. 2016;4:502-10.

17. Fonarow GC, Hsu JJ. Left ventricular ejection fraction: what is "normal"? JACC Heart Fail. 2016;4:511-3.

18. Chung AK, Das SR, Leonard D, Peshock RM, Kazi F, Abdullah SM, et al. Women have higher left ventricular ejection fractions than men independent of differences in left ventricular volume: the Dallas Heart Study. Circulation. 2006;113:1597-604.

19. Poppe KK on behalf of The EchoNoRMAL Collaboration. What is normal left ventricular ejection fraction? a global individual person data meta-analysis of the distribution across ethnicity, gender and age. Eur Heart J. 2013;34 suppl 1:1938.

20. Gebhard C, Stähli BE, Gebhard CE, Tasnady H, Zihler D, Wischnewsky MB, et al. Age- and genderdependent left ventricular remodeling. Echocardiography. 2013;30:1143-50.

21. Chuang ML, Gona P, Hautvast GL, Salton CJ, Breeuwer M, O'nnell CJ, et al. CMR reference values for left ventricular volumes, mass, and ejection fraction using computer-aided analysis: the Framingham Heart Study. J Magn Reson Imaging. 2014;39:895-900.

22. Claeys M, Roberts T, Herbots L, Verwerft J, Lefebvre K, Voigt JU, et al. Left ventricular ejection fraction: range of normal responses during semi-supine bicycle stress echocardiography. Circulation. 2018;138:A13101.

23. Veale D, Fagret D, Pepin JL, Bonnet C, Siche JP, Lévy P. Circadian changes of left ventricular ejection fraction in normal subjects. Chronobiol Int. 1994;11:200-10.

24. Gebhard C, Buechel RR, Stähli BE, Gransar H, Achenbach S, Berman DS, et al. Impact of age and sex on left ventricular function determined by coronary computed tomographic angiography: results from the prospective multicentre CONFIRM study. Eur Heart J Cardiovasc Imaging. 2017;18:990-1000.

25. Wehner GJ, Jing L, Haggerty CM, Suever JD, Leader JB, Hartzel DN, et al. Routinely reported ejection fraction and mortality in clinical practice: where does the nadir of risk lie? Eur Heart J. 2020;41:1249-57.

26. Krayenbühl HP, Bussmann WD, Turina M, Lüthy E. Is the ejection fraction an index of myocardial contractility? Cardiologia. 1968;53:1-10.

27. Sonnenblick EH. Correlation of myocardial ultrastructure and function. Circulation. 1968;38:29-44.

28. Lowes BD, GilbertEM, Abraham WT, Minobe WA, Larrabee P, Ferguson D, et al. Myocardial gene expression in dilated cardiomyopathy treated with beta-blocking agents. N Engl J Med. 2002;346:1357-65.

29. Kao DP, Lowes BD, Gilbert EM, Minobe W, Epperson LE, Meyer LK, et al. Therapeutic molecular phenotype of $\beta$-blocker-associated reverse-remodeling in nonischemic dilated cardiomyopathy. Circ Cardiovasc Genet. 2015;8:270-83.

30. Bristow MR, Kao DP, Breathett KK, Altman NL, Gorcsan J 3rd, Gill EA, et al. Structural and functional phenotyping of the failing heart: is the left ventricular ejection fraction obsolete? JACC Heart Fail. 2017;5:772-81.

31. Ikonomidis I, Aboyans V, Blacher J, Brodmann M, Brutsaert DL, Chirinos JA, et al. The role of ventriculararterial coupling in cardiac disease and heart failure: assessment, clinical implications and therapeutic interventions. A consensus document of the European Society of Cardiology Working Group on Aorta \& Peripheral Vascular Diseases, European Association of Cardiovascular Imaging, and Heart Failure Association. Eur J Heart Fail. 2019;21:402-24.

32. Bastos MB, Burkhoff D, Maly J, Daemen J, den Uil CA, Ameloot K, et al. Invasive left ventricle pressurevolume analysis: overview and practical clinical implications. Eur Heart J. 2020;41:1286-97.

33. Chen CH, Fetics B, Nevo E, Rochitte CE, Chiou KR, Ding PA, et al. Noninvasive single-beat determination of left ventricular end-systolic elastance in humans. J Am Coll Cardiol. 2001;38:2028-34.

34. Shishido T, Hayashi K, Shigemi K, Sato T, Sugimachi M, Sunagawa K. Single-beat estimation of end-systolic elastance using bilinearly approximated time-varying elastance curve. Circulation. 2000;102:1983-9. 
35. Leisman S, Burkhoff D. Use of an iPad App to simulate pressure-volume loops and cardiovascular physiology. Adv Physiol Educ. 2017;41:415-24.

36. Schafer A, Burkhoff D, Bauersachs J. Haemodynamic simulation and the effect of early left ventricular unloading in pre-shock acute coronary syndrome. ESC Heart Fail. 2019;6:457-63.

37. Kass DA, Maughan WL, Guo ZM, Kono A, Sunagawa K, Sagawa K. Comparative influence of load versus inotropic states on indexes of ventricular contractility: experimental and theoretical analysis based on pressure-volume relationships. Circulation. 1987;76:1422-36.

38. Robotham JL, Takata M, Berman M, Harasawa Y. Ejection fraction revisited. Anesthesiology. 1991;74:172-83.

39. Mihaileanu S. Left ventricular contactile reserve. In: Dorobanţu M, Ruschitzka F, Metra M, editors. Current approach to heart failure. Springer: Cham; 2016. p. 127-48.

40. Monge García MI, Jian Z, Settels JJ, Hunley C, Cecconi M, Hatib F, et al. Determinants of left ventricular ejection fraction and a novel method to improve its assessment of myocardial contractility. Ann Intensive Care. 2019;9:48.

41. Sagawa K, Suga H, Shoukas AA, Bakalar KM. End-systolic pressure/volume ratio: a new index of ventricular contractility. Am J Cardiol. 1977;40:748-53.

42. Sagawa K. The ventricular pressure-volume diagram revisited. Circ Res. 1978;43:677-87.

43. Blaudszun G, Morel DR. Relevance of the volume-axis intercept, V0, compared with the slope of endsystolic pressure-volume relationship in response to large variations in inotropy and afterload in rats. Exp Physiol. 2011;96:1179-95.

44. Kerkhof PL. Characterizing heart failure in the ventricular volume domain. Clin Med Insights Cardiol. 2015;9:11-31.

45. Li JK, Atlas G. Left Ventricle-arterial system interaction in heart failure. Clin Med Insights Cardiol. 2015;9:93-9.

46. Li JK, Kaya M, Kerkhof PLM. Quantitative cardiology and computer modeling analysis of heart failure in systole and in diastole. Comput Biol Med. 2018;103:252-61.

47. Segers P, Stergiopulos N, Westerhof N. Relation of effective arterial elastance to arterial system properties. Am J Physiol Heart Circ Physiol. 2002;282:H1041-6.

48. Mann DL, Bogaev R, Buckberg GD. Cardiac remodelling and myocardial recovery: lost in translation? Eur J Heart Fail. 2010;12:789-96.

49. Kerkhof PLM, Kuznetsova T, Ali R, Handly N. Left ventricular volume analysis as a basic tool to describe cardiac function. Adv Physiol Educ. 2018;42:130-9.

50. Kerkhof PLM, van de Ven PM, Yoo B, Peace RA, Heyndrickx GR, Handly N. Ejection fraction as related to basic components in the left and right ventricular volume domains. Int J Cardiol. 2018;255:105-10.

51. Little WC. The left ventricular dP/dtmax-end-diastolic volume relation in closed-chest dogs. Circ Res. 1985;56:808-15.

52. Meluzin J, Spinarová L, Bakala J, Toman J, Krejci J, Hude P, et al. Pulsed Doppler tissue imaging of the velocity of tricuspid annular systolic motion; a new, rapid, and non-invasive method of evaluating right ventricular systolic function. Eur Heart J. 2001;22:340-8.

53. Nikitin NP, Loh PH, Silva R, Ghosh J, Khaleva OY, Goode K, et al. Prognostic value of systolic mitral annular velocity measured with Doppler tissue imaging in patients with chronic heart failure caused by left ventricular systolic dysfunction. Heart. 2006;92:775-9.

54. Seo JS, Kim DH, Kim WJ, Song JM, Kang DH, Song JK. Peak systolic velocity of mitral annular longitudinal movement measured by pulsed tissue Doppler imaging as an index of global left ventricular contractility. Am J Physiol Heart Circ Physiol. 2010;298:H1608-15.

55. Biering-Sorensen T, Mogelvang R, de Knegt MC, Olsen FJ, Galatius S, Jensen JS. Cardiac time intervals by tissue Doppler imaging M-mode: normal values and association with established echocardiographic and 
invasive measures of systolic and diastolic function. PLoS One. 2016;11:e0153636.

56. Cikes M, Solomon SD. Beyond ejection fraction: an integrative approach for assessment of cardiac structure and function in heart failure. Eur Heart J. 2016;37:1642-50.

57. Pibarot P, Dumesnil JG. Low-flow, low-gradient aortic stenosis with normal and depressed left ventricular ejection fraction. J Am Coll Cardiol. 2012;60:1845-53.

58. Dreyfus G, Mihaileanu S. The Batista procedure. Heart. 2001;85:1-2.

59. Monin JL, Quéré JP, Monchi M, Petit H, Baleynaud S, Chauvel C, et al. Low-gradient aortic stenosis: operative risk stratification and predictors for long-term outcome: a multicenter study using dobutamine stress hemodynamics. Circulation. 2003;108:319-24.

60. Borlaug BA, Kass DA. Ventricular-vascular interaction in heart failure. Heart Fail Clin. 2008;4:23-36.

61. Zakir RM, Al-Dehneh A, Maher J, Saric M, Berkowitz RL. Right ventricular failure in patients with preserved ejection fraction and diastolic dysfunction: an underrecognized clinical entity. Congest Heart Fail. 2007;13:164-9.

62. Brand A, Bathe M, Oertelt-Prigione S, Seeland U, Rücke M, Regitz-Zagrosek V, et al. Right heart function in impaired left ventricular diastolic function: 2D speckle tracking echocardiography-based and Doppler tissue imaging-based analysis of right atrial and ventricular function. Echocardiography. 2018;35:47-55. 